\title{
Synchronization of coupled systems via parameter perturbations
}

\author{
P. J. Aston and C. M. Bird \\ Department of Mathematical and Computing Sciences, University of Surrey, Guildford GU2 5XH, United Kingdom
}

(Received 11 August 1997)

\begin{abstract}
We consider coupled identical chaotic systems. In some circumstances, the coupled systems synchronize. When this does not happen naturally, we derive methods based on small parameter perturbations which result in synchronous behavior. The perturbations are applied in the neighborhood of a fixed or periodic point in the synchronous subspace which is stable in the normal direction. By keeping iterates in the neighborhood of such points using parameter perturbations, they are naturally drawn closer to the subspace by the stable manifold of the fixed or periodic points. Different ways of varying the parameters are also considered. Methods for two-dimensional systems are first explored and then extended to higher-dimensional systems. Examples are presented to illustrate the methods. [S1063-651X(98)03903-8]

PACS number(s): $05.45 .+\mathrm{b}$
\end{abstract}

\section{INTRODUCTION}

The synchronization of chaotic systems, along with the control of chaos, has been a popular focus for recent research. The problem consists of the synchronization of two or more identical coupled chaotic oscillators such that they both exhibit identical chaotic behavior. At first sight this seems an impossible task because of the fundamental property of chaotic systems of sensitive dependence upon initial conditions. Indeed, Tang, Mees, and Chua [1] postulate that chaotic systems defy synchronization. If we were to observe the dynamics of two identical, uncoupled chaotic oscillators, each given almost identical initial conditions, eventually we would see their trajectories diverge from a synchronous (or at least, near synchronous) state to an asynchronous state. It is, of course, impossible to construct two identical chaotic oscillators in the first place, and so the problem is compounded in that we wish to "synchronize" two or more almost identical systems.

Much of the interest in this area was initiated by Pecora and Carroll [2], who demonstrated that, under certain circumstances, it is possible to synchronize the behavior of two chaotic systems by linking them with a common signal or signals. Provided that these signals are appropriately chosen, synchronization of the systems will occur spontaneously as the time development of the coupled system progresses. Yamada and Fujisaka [3] use a simple coupling technique in order to achieve synchronization of two independent oscillators. The strength of the coupling signal must be above a certain threshold for synchronization to occur naturally.

The synchronization of chaotic oscillators has a number of applications. Hayes, Grebogi, and Ott [4] looked at transmitting data securely by using a pair of coupled oscillators. Kocarev and Stojanovski [5] have also investigated the application of chaotic synchronization to secure communications. Roy and Thornburg [6] have looked at the experimental synchronization of chaotic lasers. Further recent work in this area can be found in the papers of Yu, Kwak, and Lim [7,8] and Cuomo, Oppenheim, and Strogatz [9]. For an excellent summary of some of the earlier work on synchronization, see Ogorzalek [10].

The problem we consider is that of obtaining synchroni- zation in low-dimensional systems when it would not naturally occur via the application of small parameter perturbations based on only a linear approximation to the map in the neighborhood of a fixed or periodic point. This approach is similar to the method for controlling chaos which was proposed by Ott, Grebogi, and Yorke [17] which involves stabilizing a fixed or periodic point contained in the attractor via small parameter perturbations. In this case, a fixed or periodic point in the synchronous subspace is used and parameter perturbations are employed to draw iterates close to this point. However, once this has been achieved, the control is turned off so that chaotic motion close to the invariant subspace is restored. In some cases, depending on the method of parameter perturbation, it is also possible to use perturbations to place an iterate (approximately) on the invariant subspace itself.

Lai and Grebogi [11,12] proposed a method for synchronizing two identical systems via parameter perturbations. However, in their case, there was no coupling between the two systems (other than that induced by the parameter perturbations). One system was allowed to iterate chaotically while parameter perturbations were then applied to the second system in order to keep it in step, or synchronized, with the first. The parameter perturbations were derived from a linearization about a chaotic "target" trajectory of the first system. This requires an approximation to the linearization of the system over the whole of the attractor. Once this has been obtained, the parameter perturbations can be determined at each iteration by requiring that the next iterate be moved onto the stable direction at that point. This approach has the significant disadvantage that it requires a large amount of global knowledge of the system. In particular, the linearization of the system is required over the whole of the attractor. Also parameter perturbations must be continually applied in order to maintain the synchronization. Using our approach in which there is a natural coupling between the systems but synchronization does not naturally occur, only a local approximation to the dynamics near to a periodic point is required which is much easier and cheaper to determine. Moreover parameter perturbations are only applied when the difference between the two systems grows too large and while this difference is small, parameter perturbations are not 
required so that the system behaves in its normal, unperturbed state.

Another approach for synchronizing coupled systems using parameter perturbations has been proposed by Nagai, Hua, and Lai [13]. Their method consists of making parameter perturbations at every iteration. The criterion for determining the perturbations is that one component of the normal variables should be zero at the next iteration. It is also important with this method that the parameter chosen for perturbations has an effect on the normal variables and not just on those in the invariant subspace. Their method is illustrated with a two-dimensional map which has a onedimensional invariant subspace. It is significant, however, that this map is not associated with a synchronization problem. There are a number of drawbacks with this approach. The first is that it requires that the map be known although an alternative ad hoc method is also suggested which does not require the map to be known. This is again because global information is required as perturbations are made at every iteration. Since our method is essentially local, with perturbations only being made when iterates are close to a periodic point, the map does not need to be known since a local approximation near to the periodic points can easily be obtained. The requirement that the parameter perturbs the normal variables implies that it will also perturb the invariant subspace, as is the case in their example. However, in some examples of synchronization problems, this is not the case and so their method would not work. We propose methods which will work in this situation by keeping iterates near to a periodic point which is attracting in the normal direction. Thus this natural attraction of the system is used to draw iterates in close to the invariant subspace.

Ashwin, Buescu, and Stewart $[14,15]$ note that the problem of the synchronization of identical systems is just one example of a very general situation in which the same issues arise. The essential ingredients are a dynamical system with an invariant subspace. The stability of the chaotic motion in the invariant subspace with respect to transverse perturbations is determined by normal Lyapunov exponents. Symmetry provides a natural setting for such invariant subspaces since fixed point subspaces are always invariant. Coupled identical oscillators have an invariant subspace corresponding to the synchronized state.

In Sec. II we consider two coupled one-dimensional maps and different methods for using parameter perturbations to obtain synchronization are proposed. An example illustrates the methods. In Sec. III the methods are extended to higherdimensional systems and an example of coupled Duffing systems is used to illustrate the use of the methods. Finally, applications for this approach are considered in Sec. IV.

\section{TWO-DIMENSIONAL SYSTEMS}

We consider two-dimensional systems of iterated maps with a one-dimensional invariant subspace. One method of generating such problems is by coupling two onedimensional maps. The invariant subspace then corresponds to the synchronized state.

Consider the system of coupled maps given by

$$
X_{n+1}=f\left(X_{n}, p^{*}+\delta p_{n}\right)+c\left(Y_{n}-X_{n}\right),
$$

$$
Y_{n+1}=f\left(Y_{n}, p^{*}+\delta q_{n}\right)+c\left(X_{n}-Y_{n}\right),
$$

where $f: \mathbb{R} \times \mathbb{R} \rightarrow \mathbb{R}$ and $c \in \mathbb{R}$ is the coupling parameter. We assume that perturbations can be made independently in each of the two systems and so there are two perturbation parameters $\delta p_{n}$ and $\delta q_{n}$. We suppose that when $\delta p_{n}=\delta q_{n}=0$, the dynamics in the invariant subspace defined by $X=Y$ is chaotic with a positive normal Lyapunov exponent so that the basin of attraction of this synchronized state in the whole space has measure zero [14]. We also assume that the dynamics of the coupled system is chaotic in the whole space and that the attractor is "stuck on" to the invariant subspace so that the iterates spend a long time close to the invariant subspace [16]. Our aim is to use the parameter perturbations $\delta p_{n}$ and $\delta q_{n}$ to synchronize the coupled system. In practice, this means keeping the iterates very close to the invariant subspace for long time periods. The methods that we use are essentially "local" methods in that they require information only in a small neighborhood of a fixed or periodic point which is contained in the invariant subspace. This is in contrast to the methods of Lai and Grebogi $[11,12]$ and Nagai, Hua, and Lai [13] where global information about the attractor is required.

When $\delta p_{n}=\delta q_{n}$, Eqs. (1) have a $\mathbf{Z}_{2}$ symmetry defined by

$$
S\left[\begin{array}{c}
X_{n} \\
Y_{n}
\end{array}\right]=\left[\begin{array}{c}
Y_{n} \\
X_{n}
\end{array}\right] .
$$

It is convenient to perform a change of variables so that the invariant subspace is one of the coordinate axes. Thus we define

$$
x_{n}=\frac{X_{n}+Y_{n}}{2}, \quad y_{n}=\frac{X_{n}-Y_{n}}{2},
$$

and Eqs. (1) become

$$
\begin{aligned}
x_{n+1}= & \frac{1}{2}\left[f\left(x_{n}+y_{n}, p^{*}+\delta p_{n}\right)+f\left(x_{n}-y_{n}, p^{*}+\delta q_{n}\right)\right], \\
y_{n+1}= & \frac{1}{2}\left[f\left(x_{n}+y_{n}, p^{*}+\delta p_{n}\right)-f\left(x_{n}-y_{n}, p^{*}+\delta q_{n}\right)\right] \\
& -2 c y_{n} .
\end{aligned}
$$

The symmetry of these equations is defined by

$$
S\left[\begin{array}{l}
x \\
y
\end{array}\right]=\left[\begin{array}{c}
x \\
-y
\end{array}\right],
$$

and the one-dimensional invariant subspace is defined by $y$ $=0$.

\section{A. Methods for synchronization}

Since the attractor is stuck onto the invariant subspace, this implies that there are periodic points in the invariant subspace which are attracting in the normal direction and are therefore saddles in the two-dimensional space. A first simplistic approach to using parameter perturbations to synchronize the coupled system essentially consists of using the Ott, Grebogi, and Yorke method [17] for controlling chaos by stabilizing a fixed or periodic point contained in the attractor. Thus a fixed point is found in the invariant subspace which is 
attracting in the normal direction. Once iterates come near to this point, parameter perturbations are used to move iterates onto the stable manifold and so they are attracted to the invariant subspace. When it is considered that an iterate is sufficiently close to the subspace, the parameter perturbations can be turned off, allowing the iterates to wander chaotically close to the invariant subspace. When the iterates start to move away from the subspace but come close to the stable manifold of the fixed point, the parameter perturbations can again be activated. Since the attractor is stuck onto its invariant subspace, this also means that the iterates often come close to the subspace and this means that data near to the fixed point in the invariant subspace can be collected which enables the linearized dynamics required for the control method to be estimated.

Since the aim of the perturbations in this context is to attract iterates to the invariant subspace rather than to the fixed point, it seems reasonable to consider whether a perturbation could be chosen with the aim of obtaining $y_{n+1}=0$ rather than simply aiming for the stable manifold and letting the iterates slowly drift in. However, if both systems are perturbed identically so that $\delta p_{n}=\delta q_{n}$, then the derivative of the coupled system (3) with respect to $\delta p_{n}$ evaluated at a fixed point $\left(x^{*}, p^{*}\right)$ in the invariant subspace is $\mathbf{w}$ $=\left[f_{p}\left(x^{*}, p^{*}\right), 0\right]^{T}$. Now iterates move in the direction of the vector $\mathbf{w}$ when the parameter is perturbed and so this implies that the iterates move parallel to the invariant subspace. Thus it is not possible to place an iterate on the invariant subspace directly in this case.

Once the iterates have come close to the invariant subspace, the intention is that they then continue chaotically close to the invariant subspace. However, since they have been attracted to the stable manifold of a fixed point by the parameter perturbations, the iterates are likely to stay near to the fixed point initially. To speed up the escape from the fixed point, a final parameter perturbation could be used to move the iterate away from the fixed point so that chaotic motion is quickly restored.

An alternative approach is to start with an iterate which is close to the stable manifold of the fixed point in the invariant subspace and use parameter perturbations to fix the value of $x$. Since it is assumed that the initial iterate is close to the stable manifold, the iterates will again be attracted to the invariant subspace. However, a final kick to move the iterates away from the fixed point is not required since they will not be very close to that fixed point.

The linearization of Eq. (3) about a fixed point in the invariant subspace has the form

$$
\left[\begin{array}{c}
\delta x_{n+1} \\
\delta y_{n+1}
\end{array}\right]=\left[\begin{array}{cc}
\lambda_{u} & 0 \\
0 & \lambda_{s}
\end{array}\right]\left[\begin{array}{l}
\delta x_{n} \\
\delta y_{n}
\end{array}\right]+\left[\begin{array}{l}
w \\
0
\end{array}\right] \delta p_{n}
$$

where $\delta x_{n}=x_{n}-x^{*}, \delta y_{n}=y_{n}, \lambda_{s}$ and $\lambda_{u}$ are the stable and unstable eigenvalues associated with the saddle fixed point, and $w=f_{p}\left(x^{*}, p^{*}\right)$. Note that the matrix is diagonal due to the reflectional symmetry in the problem. Suppose that an iterate $\left(x_{n}, y_{n}\right)=(\hat{x}, \hat{y})$ comes close to the stable manifold of the fixed point, that is $\hat{x} \in R_{\delta}=\left[x^{*}-\delta, x^{*}+\delta\right]$ for some small $\delta>0$. If the aim is to have $\delta x_{n+1}=\hat{x}-x^{*}$ also, then from the first equation of Eq. (5), the required parameter perturbation is given by

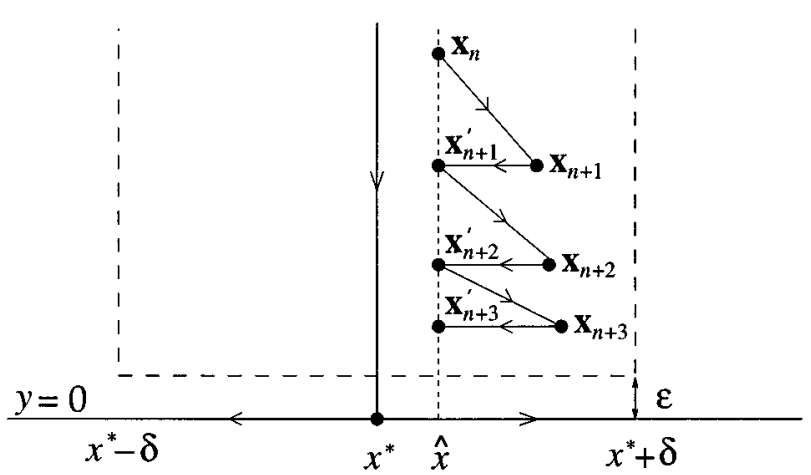

FIG. 1. Using parameter perturbations to keep the $x$ coordinate fixed in the vicinity of the fixed point.

$$
\delta p_{n}=\frac{\hat{x}-x^{*}-\lambda_{u}\left(x_{n}-x^{*}\right)}{w} .
$$

Note that at the first iteration, $x_{n}=\hat{x}$ but this will not hold precisely for subsequent iterations since the parameter perturbation is determined from approximate linear dynamics about the fixed point. Since the normal dynamics is independent of the parameter perturbations, it is simply given by the second equation of Eq. (5). Thus

$$
\delta y_{n+1}=\lambda_{s} \delta y_{n}
$$

and so there is contraction in the $y$ variable towards the invariant subspace and the rate of contraction is determined by the stable eigenvalue $\lambda_{s}$. This process is continued until an iterate is within a distance $\epsilon$ of the invariant subspace for some small $\epsilon>0$. The parameter perturbations are then turned off and the chaotic motion near to the invariant subspace is resumed (see Fig. 1).

We have considered how perturbations can be used to synchronize coupled systems when the perturbations to both systems are the same, i.e., $\delta p_{n}=\delta q_{n}$. If this condition does not hold, then the equations have $\mathbf{Z}_{2}$ symmetry in their unperturbed state $\left(\delta p_{n}=\delta q_{n}=0\right)$ but a perturbation with $\delta p_{n}$ $\neq \delta q_{n}$ corresponds to a symmetry breaking perturbation of the equations. However, this can be advantageous since parameter perturbations in this case do not move the iterates parallel to the invariant subspace. Thus it is now possible to choose a perturbation to put an iterate on the invariant subspace, rather than waiting for the orbit to drift down the stable manifold of a fixed point. Of course, an approximation to the linear dynamics is still required and we obtain this in the usual way in the neighborhood of a fixed point.

The equations in the transformed coordinates with $\delta p_{n}$ $\neq 0$ and $\delta q_{n}=0$ are given by

$$
\begin{gathered}
x_{n+1}=\frac{1}{2}\left[f\left(x_{n}+y_{n}, p^{*}+\delta p_{n}\right)+f\left(x_{n}-y_{n}, p^{*}\right)\right], \\
y_{n+1}=\frac{1}{2}\left[f\left(x_{n}+y_{n}, p^{*}+\delta p_{n}\right)-f\left(x_{n}-y_{n}, p^{*}\right)\right]-2 c y_{n}
\end{gathered}
$$

and linearizing these equations about a fixed point $\left(x^{*}, 0\right)$ with $p=p^{*}$ gives

$$
\left[\begin{array}{l}
\delta x_{n+1} \\
\delta y_{n+1}
\end{array}\right]=\left[\begin{array}{cc}
\lambda_{u} & 0 \\
0 & \lambda_{s}
\end{array}\right]\left[\begin{array}{l}
\delta x_{n} \\
\delta y_{n}
\end{array}\right]+\frac{1}{2}\left[\begin{array}{l}
w \\
w
\end{array}\right] \delta p_{n} .
$$


Now the dynamics in the transverse direction is given by

$$
\delta y_{n+1}=\lambda_{s} \delta y_{n}+\frac{1}{2} w \delta p_{n} .
$$

To place an iterate on the invariant subspace, we require that $\delta y_{n+1}=y_{n+1}=0$ and so the required perturbation is

$$
\delta p_{n}=-\frac{2 \lambda_{s} \delta y_{n}}{w} .
$$

If the value of $\left|\delta p_{n}\right|$ is larger than the maximum allowed perturbation $\delta p_{\max }$, then some perturbations which move the iterates onto the stable manifold of the fixed point can first be performed until an iterate is close enough to be placed onto the invariant subspace with a sufficiently small perturbation.

One problem associated with both methods considered in this section is that the time spent away from the neighborhood of the fixed point in which parameter perturbations can be applied may be long, in which case synchronization may be lost. The simple solution to this problem is to use a higher period point in the invariant subspace which is attracting in the normal direction. Then, once the iterates start to move away from the invariant subspace, there will only be a short interval until an iterate falls near to one of the periodic points and then parameter perturbations can be applied to draw the orbit back towards the invariant subspace again.

\section{B. Example}

The form of coupled one-dimensional maps is rather restrictive and so we consider a two-dimensional system which has a one-dimensional invariant subspace given by $y=0$. In particular, we consider the equations

$$
\begin{gathered}
x_{n+1}=4 x_{n}\left[1-(1+p) x_{n}\right]+y_{n}^{2}\left(2.8 x_{n}^{2}-2.8 x_{n}+0.5\right), \\
y_{n+1}=y_{n}\left(c_{0} e^{\left.-y_{n}^{2}+c_{1} x_{n}+c_{2} x_{n}^{2}+c_{3} x_{n}^{3}\right),}\right.
\end{gathered}
$$

where

$$
c_{0}=0.9557, \quad c_{1}=6.277, \quad c_{2}=-16.246, \quad c_{3}=9.846 .
$$

The nominal value of $p$ is chosen to be $p^{*}=0$ and the iteration in the invariant subspace is then given by

$$
x_{n+1}=F_{0}\left(x_{n}\right)=4 x_{n}\left(1-x_{n}\right),
$$

for which the invariant density on the interval $I=[0,1]$ is given by

$$
\nu(x)=\frac{1}{\pi \sqrt{x(1-x)}} .
$$

The normal Lyapunov exponent is then

$$
\sigma=\int_{0}^{1} \ln \left(c_{0}+c_{1} x_{n}+c_{2} x_{n}^{2}+c_{3} x_{n}^{3}\right) \nu(x) d x=0.01706,
$$

and since it is positive, the chaotic motion in the invariant subspace is unstable with respect to almost all normal perturbations. There is a fixed point of $F_{0}$ at $x=0.75$ and the eigenvalues of the linearization of Eqs. (6) at this point are

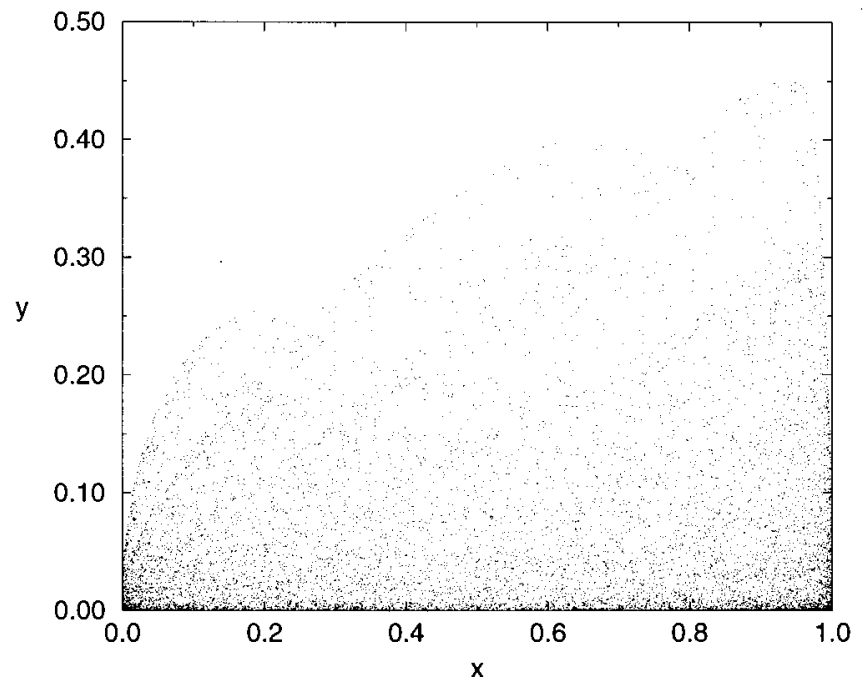

FIG. 2. Uncontrolled chaotic attractor.

$\lambda_{u}=-2$ and $\lambda_{s}=0.6788$. We choose the box near to this fixed point to have $\delta=0.05$ and $\epsilon=0.0001$ so that $R_{\delta}$ $=[0.7,0.8]$.

The uncontrolled chaotic attractor is shown in Fig. 2. Using parameter perturbations in order to fix the value of $x$ once an iterate falls in the control region to obtain synchronization (see Fig. 1) gives the attractor shown in Fig. 3 which contains 10000 iterations. Vertical lines can clearly be seen when $x_{n} \in[0.7,0.8]$ arising from the control mechanism. Not all the iterates are shown in this figure but the largest value of $y_{n}$ is 0.0162 which is small when compared with the uncontrolled attractor. The maximum parameter perturbation required is 0.0647 so small perturbations are sufficient to achieve synchronization. If the iteration is left to run for longer time periods, then occasionally synchronization is lost for short periods. However, we have constructed this example to work by using a fixed point of the map. Using a higher period orbit would give a method for which synchro-

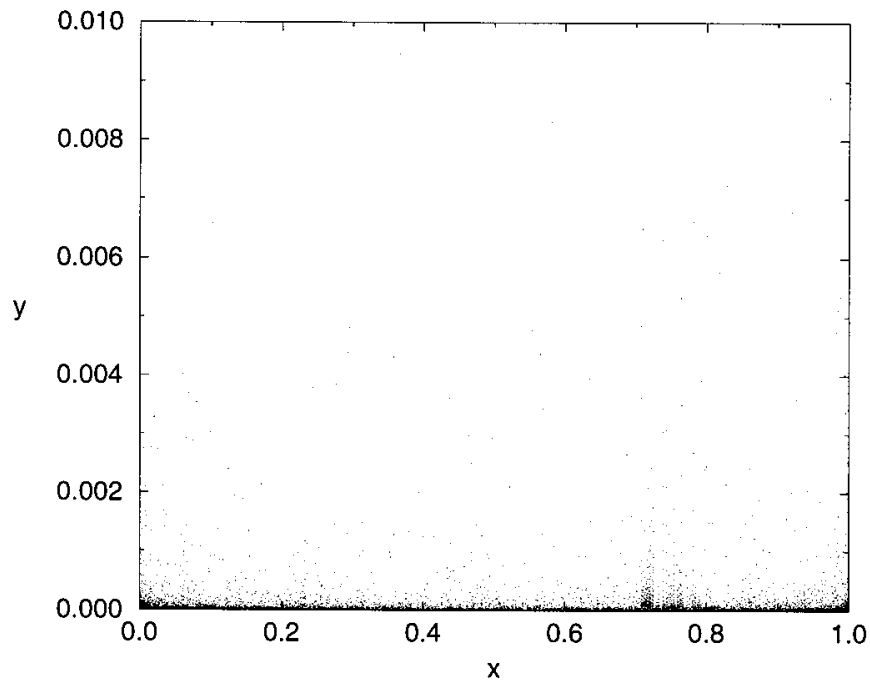

FIG. 3. Synchronized attractor. 
nization could be achieved for very long times. Better synchronization can also be obtained by decreasing $\epsilon$.

\section{HIGHER-DIMENSIONAL SYSTEMS}

In order to extend the synchronization methods for use on a general $N$-dimensional problem, we consider synchronization in four-dimensional systems. The extension to higher dimensions should then be apparent. In particular, we consider the coupling of a pair of two-dimensional maps and extend the theory of Sec. II to this case. We then apply these methods to the coupling of a pair of Duffing oscillators by working with the Poincaré map.

Consider a pair of coupled two-dimensional iterated maps

$$
\left[\begin{array}{l}
\mathbf{X}_{n+1} \\
\mathbf{Y}_{n+1}
\end{array}\right]=\left[\begin{array}{l}
\mathbf{f}\left(\mathbf{X}_{n}, p^{*}+\delta p_{n}\right) \\
\mathbf{f}\left(\mathbf{Y}_{n}, p^{*}+\delta q_{n}\right)
\end{array}\right]+B\left[\begin{array}{l}
\mathbf{X}_{n} \\
\mathbf{Y}_{n}
\end{array}\right]
$$

where $f: \mathbb{R}^{2} \times \mathbb{R} \rightarrow \mathbb{R}^{2}$ and $B$ is a $4 \times 4$ matrix which describes the linear coupling. The matrix $B$ can be expressed in the form $B=C \otimes D$ where $C$ and $D$ are $2 \times 2$ matrices. The matrix $D$ describes the coupling arrangement between different components of the two systems while $C$ describes the coupling connections and the strength of the couplings [18]. We assume that there is two-way coupling between the two systems and so we can write

$$
C=\left[\begin{array}{cc}
-c & c \\
c & -c
\end{array}\right], \quad D=\left[\begin{array}{ll}
d_{11} & d_{12} \\
d_{21} & d_{22}
\end{array}\right]
$$

Using the transformation of variables

$$
\mathbf{x}_{n}=\frac{\mathbf{X}_{n}+\mathbf{Y}_{n}}{2}, \quad \mathbf{y}_{n}=\frac{\mathbf{X}_{n}-\mathbf{Y}_{n}}{2}
$$

in Eq. (7) yields the system

$$
\begin{aligned}
{\left[\begin{array}{l}
\mathbf{x}_{n+1} \\
\mathbf{y}_{n+1}
\end{array}\right]=} & \frac{1}{2}\left[\begin{array}{l}
\mathbf{f}\left(\mathbf{x}_{n}+\mathbf{y}_{n}, p^{*}+\delta p_{n}\right)+\mathbf{f}\left(\mathbf{x}_{n}-\mathbf{y}_{n}, p^{*}+\delta q_{n}\right) \\
\mathbf{f}\left(\mathbf{x}_{n}+\mathbf{y}_{n}, p^{*}+\delta p_{n}\right)-\mathbf{f}\left(\mathbf{x}_{n}-\mathbf{y}_{n}, p^{*}+\delta q_{n}\right)
\end{array}\right] \\
& +B^{\prime}\left[\begin{array}{l}
\mathbf{x}_{n} \\
\mathbf{y}_{n}
\end{array}\right]
\end{aligned}
$$

where

$$
B^{\prime}=C^{\prime} \otimes D, \quad C^{\prime}=\left[\begin{array}{cc}
0 & 0 \\
0 & -2 c
\end{array}\right] .
$$

We now consider different methods of perturbing the parameters in Eq. (8) which result in approximately synchronous behavior. The two-dimensional synchronous subspace is defined by $\mathbf{y}_{n}=\mathbf{0}$. For all the methods we consider we assume that a fixed point $\left(\mathbf{x}^{*}, p^{*}\right)$ exists within the synchronous subspace which is a saddle when restricted to this subspace. The methods can of course be generalized to deal with periodic points but we consider only fixed points for the sake of clarity.

\section{A. Perturbing both systems simultaneously}

We begin by setting $\delta q_{n}=\delta p_{n}$, so that both systems are perturbed in an identical manner. The linearization of Eq. (8) about the fixed point in the synchronous subspace then has the form

$$
\left[\begin{array}{l}
\delta \mathbf{x}_{n+1} \\
\delta \mathbf{y}_{n+1}
\end{array}\right]=\left[\begin{array}{cc}
M_{S} & 0 \\
0 & M_{N}
\end{array}\right]\left[\begin{array}{l}
\delta \mathbf{x}_{n} \\
\delta \mathbf{y}_{n}
\end{array}\right]+\delta p_{n}\left[\begin{array}{c}
\mathbf{w} \\
\mathbf{0}
\end{array}\right],
$$

where $\mathbf{w}=\mathbf{f}_{p}\left(\mathbf{x}^{*}, p^{*}\right)$. Again $\mathbf{w}$ lies parallel to the synchronous subspace and so the Ott, Grebogi, and Yorke method for stabilizing the fixed point can only be applied if both the eigenvalues of the matrix $M_{N}$ are less than one in modulus since parameter perturbations have no effect on the normal dynamics. This implies that the fixed point must have a three-dimensional stable manifold and a one-dimensional unstable manifold. Parameter perturbations can then be used to place iterates on the three-dimensional stable manifold in which case they will be attracted to the fixed point in the synchronous subspace. Once the fixed point has been stabilized, an additional perturbation can be applied to quickly restore synchronous chaotic behavior.

This method is rather restrictive in that a fixed or periodic point with a three-dimensional stable manifold is necessary in order to apply control. Such points may be uncommon or may not even exist for a particular system.

\section{B. Perturbing one system}

Suppose that perturbations are made only to one system so that $\delta q_{n}=0$. In this case, the derivative vector with respect to $\delta p_{n}$ is no longer parallel to the synchronous subspace and is given by

$$
\frac{1}{2}\left[\begin{array}{l}
\mathbf{w} \\
\mathbf{w}
\end{array}\right]
$$

The restriction of requiring the fixed point to have a threedimensional stable manifold can now be lifted. Suppose that $M_{N}$ has eigenvalues $\lambda_{s}^{N}$ and $\lambda_{u}^{N}$ where $\left|\lambda_{s}^{N}\right|<1<\left|\lambda_{u}^{N}\right|$ so that the fixed point has a two-dimensional stable manifold. A pair of parameter perturbations could then be used to place an iterate onto the two-dimensional stable manifold of the fixed point. Alternatively, a pair of parameter perturbations could be applied to place an iterate onto the synchronous subspace when this is possible. Iterating the normal component of the linearization twice gives

$$
\delta \mathbf{y}_{n+2}=M_{N}^{2} \delta \mathbf{y}_{n}+\frac{1}{2} M_{N} \mathbf{w} \delta p_{n}+\frac{1}{2} \mathbf{w} \delta p_{n+1}
$$

We assume that $\mathbf{w}$ is not an eigenvector of $M_{N}$ so that $M_{N} \mathbf{w}$ and $\mathbf{w}$ are linearly independent vectors. Let $\mathbf{f}_{1}$ and $\mathbf{f}_{2}$ be vectors orthogonal to $\mathbf{w}$ and $M_{N} \mathbf{w}$, respectively. To place an iterate onto the synchronous subspace we require that $\delta \mathbf{y}_{n+2}=\mathbf{0}$ which is equivalent to requiring that $\mathbf{f}_{1}^{T} \delta \mathbf{y}_{n+2}=0$ and $\mathbf{f}_{2}^{T} \delta \mathbf{y}_{n+2}=0$. Thus from Eq. (10) we obtain

$$
\delta p_{n}=-\frac{2 \mathbf{f}_{1}^{T} M_{N}^{2} \delta \mathbf{y}_{n}}{\mathbf{f}_{1}^{T} M_{N} \mathbf{w}}
$$




$$
\delta p_{n+1}=-\frac{2 \mathbf{f}_{2}^{T} M_{N}^{2} \delta \mathbf{y}_{n}}{\mathbf{f}_{2}^{T} \mathbf{w}} .
$$

These perturbations can be applied as soon as $\left|\delta p_{n}\right|$ $<\delta p_{\max }$ and $\left|\delta p_{n+1}\right|<\delta p_{\max }$. Parameter perturbations can then be turned off until the iterates return to a neighborhood of the fixed point at some later time.

An alternative to using two successive perturbations of a single parameter to counteract the effects of the twodimensional unstable manifold is to use two parameters. If the parameters are $p^{1}$ and $p^{2}$ with $\mathbf{w}_{i}$ $=\mathbf{f}_{p^{i}}\left(\mathbf{x}^{*},\left(p^{1}\right)^{*},\left(p^{2}\right)^{*}\right), \quad i=1,2$, then the normal linearized map around the fixed point is

$$
\delta \mathbf{y}_{n+1}=M_{N} \delta \mathbf{y}_{n}+\frac{1}{2} \mathbf{w}_{1} \delta p_{n}^{1}+\frac{1}{2} \mathbf{w}_{2} \delta p_{n}^{2} .
$$

Let $\mathbf{g}_{1}$ and $\mathbf{g}_{2}$ be vectors satisfying $\mathbf{g}_{i}^{T} \mathbf{w}_{j}=0$ if $i \neq j$. Then from Eq. (11), we obtain the parameter perturbations

$$
\begin{gathered}
\delta p_{n}^{1}=-\frac{2 \mathbf{g}_{1}^{T} M_{N} \delta \mathbf{y}_{n}}{\mathbf{g}_{1}^{T} \mathbf{w}_{1}}, \\
\delta p_{n}^{2}=-\frac{2 \mathbf{g}_{2}^{T} M_{N} \delta \mathbf{y}_{n}}{\mathbf{g}_{2}^{T} \mathbf{w}_{2}},
\end{gathered}
$$

which can be used to place an iterate onto the synchronous subspace provided that $\left|\delta p_{n}^{i}\right|<\delta p_{\text {max }}^{i}, i=1,2$.

\section{Example}

We now apply the methods discussed in the preceding section to the four-dimensional Poincaré map derived from two coupled Duffing equations. Duffing's equation is given by

$$
\frac{d^{2} X}{d t^{2}}+K \frac{d X}{d t}+X^{3}-X=A \cos \omega t
$$

and sampling the solution of this equation once per period of the forcing term gives a two-dimensional Poincare map. If we have a simple linear coupling of two such oscillators with the coupling defined by

$$
C=\left[\begin{array}{cc}
-c & c \\
c & -c
\end{array}\right], \quad D=\left[\begin{array}{ll}
0 & 0 \\
0 & 1
\end{array}\right],
$$

then we obtain the first order system

$$
\begin{gathered}
\dot{X}_{1}=X_{2}, \\
\dot{X}_{2}=-K_{1} X_{2}-X_{1}^{3}+X_{1}+A \cos \omega t+c\left(Y_{2}-X_{2}\right), \\
\dot{Y}_{1}=Y_{2}, \\
\dot{Y}_{2}=-K_{2} Y_{2}-Y_{1}^{3}+Y_{1}+A \cos \omega t+c\left(X_{2}-Y_{2}\right) .
\end{gathered}
$$

We define the change of variables

$$
x_{1}=\frac{X_{1}+Y_{1}}{2}, \quad x_{2}=\frac{X_{2}+Y_{2}}{2},
$$

$$
y_{1}=\frac{X_{1}-Y_{1}}{2}, \quad y_{2}=\frac{X_{2}-Y_{2}}{2},
$$

and take $K$ as the control parameter in each system so that

$$
K_{1}=K+\delta K_{1}, \quad K_{2}=K+\delta K_{2} .
$$

The equations then become

$$
\begin{gathered}
\dot{x}_{1}=x_{2}, \\
\dot{x}_{2}=-K x_{2}-\frac{1}{2} \delta K_{1}\left(x_{2}+y_{2}\right)-\frac{1}{2} \delta K_{2}\left(x_{2}-y_{2}\right)-\left(x_{1}^{3}+3 x_{1} y_{1}^{2}\right) \\
+x_{1}+A \cos \omega t, \\
\dot{y}_{1}=y_{2}, \\
\dot{y}_{2}=-K y_{2}-\frac{1}{2} \delta K_{1}\left(x_{2}+y_{2}\right)+\frac{1}{2} \delta K_{2}\left(x_{2}-y_{2}\right)-\left(y_{1}^{3}+3 x_{1}^{2} y_{1}\right) \\
+y_{1}-2 c y_{1} .
\end{gathered}
$$

Note that the coupling only appears in the last equation and the forcing term only in the second equation. Sampling the solutions of these equations once per period of the forcing then gives rise to a four-dimensional Poincare map. When $\delta K_{1}=\delta K_{2}=0$, the equations have a reflectional symmetry and a two-dimensional synchronous subspace defined by $y_{1}$ $=y_{2}=0$. Thus the linearization of the Poincare map at a fixed point in the subspace will have the same structure as in Eq. (9) when $\delta K_{1}=\delta K_{2}$.

Since the map is not known in closed form, the calculation of $M, \mathbf{w}$, and $\mathbf{x}^{*}$ must be done using regression. In order to obtain reliable results, one has to carry out this regression procedure with some care. The two blocks $M_{S}$ and $M_{N}$ of the linearization can be calculated separately. Clearly $M_{S}$ can be calculated by finding the linearization around the fixed point in the invariant subspace. The value of the fixed point can also be found from the regression. By collecting data near to the fixed point from the attractor in the whole space, the matrix $M_{N}$ can be found using regression by considering only the normal variables $y_{1}$ and $y_{2}$ since the linearized dynamics decouples. It is not necessary to estimate the value of the fixed point in the normal variables since it is known that it occurs at $y_{1}=y_{2}=0$. The two-dimensional vector $\mathbf{w}$ can be determined by considering the effect of a perturbation in the parameter on the dynamics in the invariant subspace. The full four-dimensional vector can then be constructed from this, depending on the particular type of parameter perturbations which are employed (see [19] for more details).

We take parameter values for the coupled Duffing oscillators of $K=0.1, A=3.0$, and $\omega=0.2$. Synchronization of the coupled oscillators occurs when $c>0.715$. When $c \simeq 0.715$, a blowout bifurcation occurs and the dynamics are no longer confined to the synchronous subspace for smaller values of $c$.

A projection of the Poincare section where $y_{1}$ is plotted against $x_{1}$ is shown in Fig. 4. A saddle fixed point is located at $\left(x_{1}, x_{2}, y_{1}, y_{2}\right)=(1.401,1.668,0.0,0.0)$ and at that point 


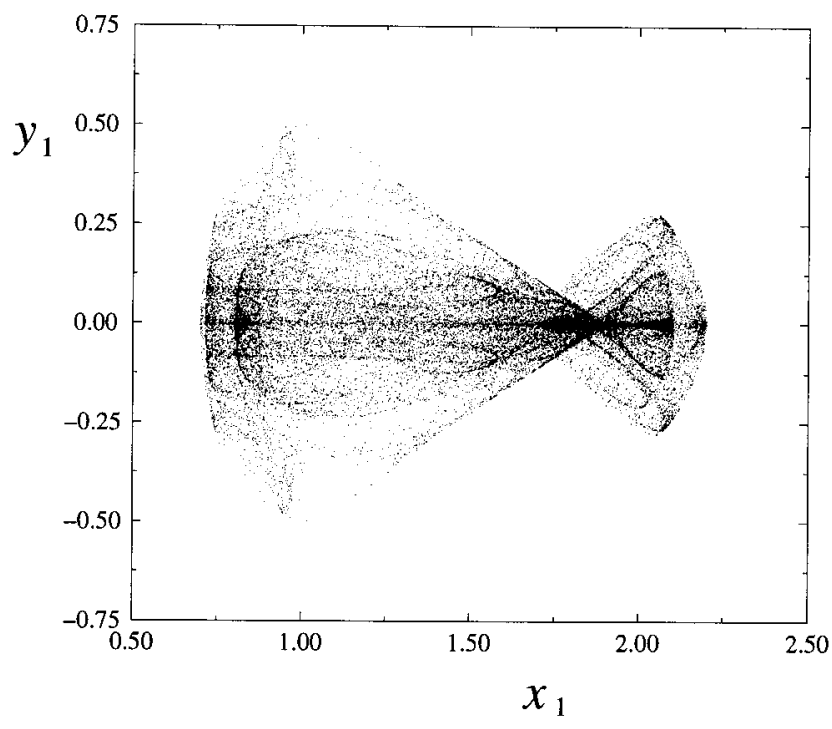

FIG. 4. Poincaré section of the coupled Duffing system with $K=0.1, A=3.0, \omega=0.2$, and $c=0.68$.

$$
\begin{gathered}
M_{S} \simeq\left[\begin{array}{ll}
-5.250 & 3.947 \\
-3.014 & 2.244
\end{array}\right], \quad M_{N} \simeq\left[\begin{array}{ll}
-1.379 & -0.314 \\
1.856 & 0.386
\end{array}\right], \\
\mathbf{w} \simeq\left[\begin{array}{r}
-21.759 \\
-18.748
\end{array}\right]
\end{gathered}
$$

The eigenvalues of $M_{S}$ are -2.967 and -0.039 while those of $M_{N}$ are -0.939 and -0.054 . Thus the fixed point has a three-dimensional stable manifold and this permits the use of the Ott, Grebogi, and Yorke method to stabilize the fixed point in order to induce synchronous behavior. Convergence of the controlled iterations will be governed by the largest of the stable eigenvalues (in absolute value) and hence will be quite slow. Parameter perturbations are activated when iterates fall within a distance of 0.1 of the fixed point. With a randomly chosen initial condition, a short transient is seen before the system is brought under control. Perturbations are applied until iterates lie within a distance of $1 \times 10^{-8}$ of the fixed point and then turned off, with the exception of a randomly chosen chaos restoring perturbation. The iterates then wander chaotically close to the synchronous subspace for a while before they begin to wander away from the vicinity of the synchronous subspace. Control is then reapplied when iterates fall close to the fixed point to maintain the dynamics close to that subspace. However, chaotic behavior can be suppressed for quite some time while control is reapplied due to the slow convergence of the method. Figure 5 shows a projection of the dynamics in the transverse direction. The dynamics remain close to the synchronous subspace for over 4000 iterations of the Poincare map, although much of this time is spent near to the fixed point during the control process.

Fixed points with a three-dimensional stable manifold prove difficult to find within the coupled Duffing system and then, as in the above example, convergence of the control iteration is often very slow. Thus this method is of little practical use. Saddles with two stable directions are far more numerous, thus suggesting that the perturbation of one system would be more preferable. We now take parameter val-

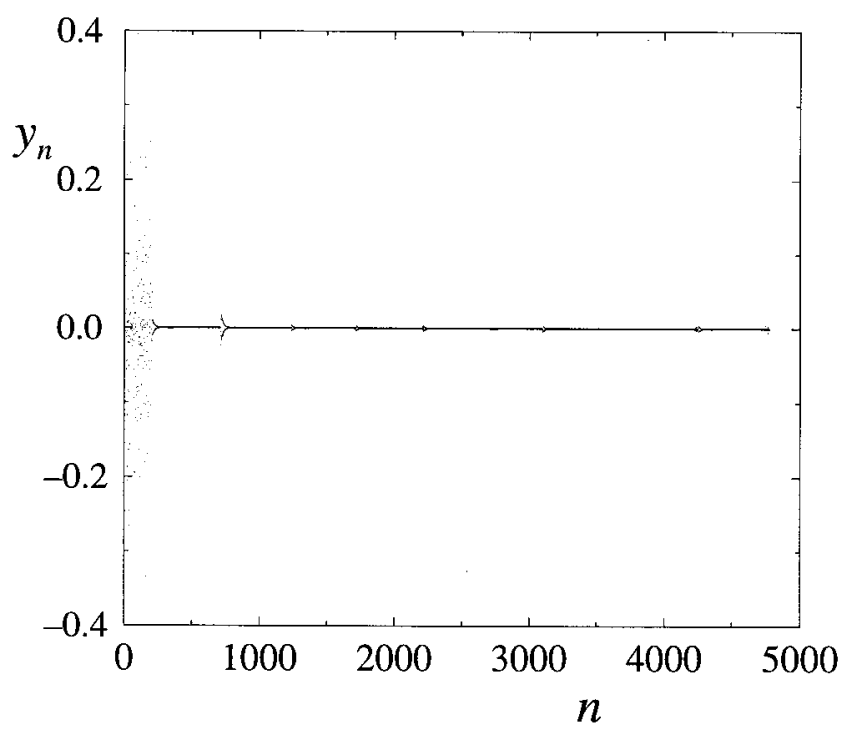

FIG. 5. A projection of the transverse dynamics under control.

ues of $K=0.5, A=2.5, \omega=2.6$, and $c=0.13$. A projection of the Poincaré section in this case is shown in Fig. 6. For these parameter values, no low order periodic points lying within the synchronous subspace with a three-dimensional stable manifold could be found. Thus the method of the simultaneous perturbation of both systems cannot be implemented on this system. A period 2 point was located at $\left(x_{1}, x_{2}, y_{1}, y_{2}\right)=(0.174,-0.052,0,0)$ and at that point, the linearization of the twice iterated map gives

$$
\begin{gathered}
M_{S} \simeq\left[\begin{array}{rr}
-1.013 & -1.524 \\
-0.826 & -1.336
\end{array}\right], \\
M_{N} \simeq\left[\begin{array}{rr}
-0.823 & -0.934 \\
-0.627 & -0.743
\end{array}\right], \quad \mathbf{w} \simeq\left[\begin{array}{l}
1.562 \\
1.738
\end{array}\right] .
\end{gathered}
$$

The eigenvalues of $M_{S}$ are -0.041 and -2.308 while those of $M_{N}$ are -0.017 and -1.549 . Since the two stable eigenvalues are small, convergence to the fixed point will occur relatively rapidly under control. Again, perturbations are ac-

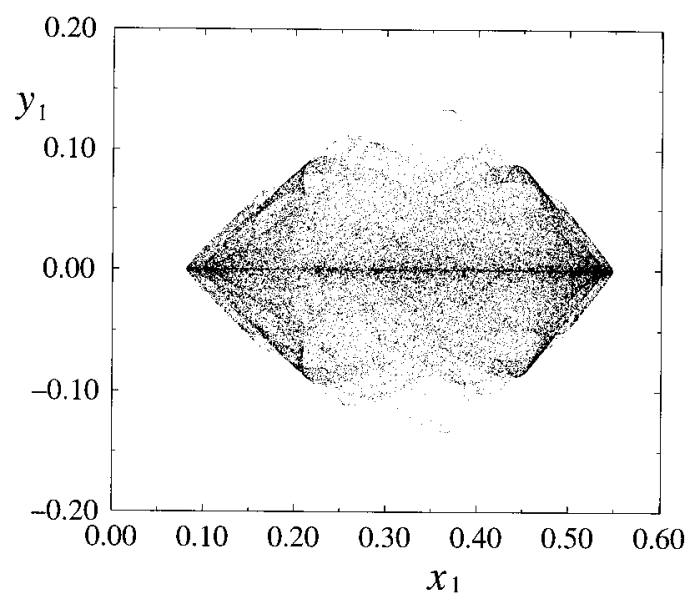

FIG. 6. Poincare section of the coupled Duffing system with $K=0.5, A=2.5, \omega=2.6$, and $c=0.13$. 


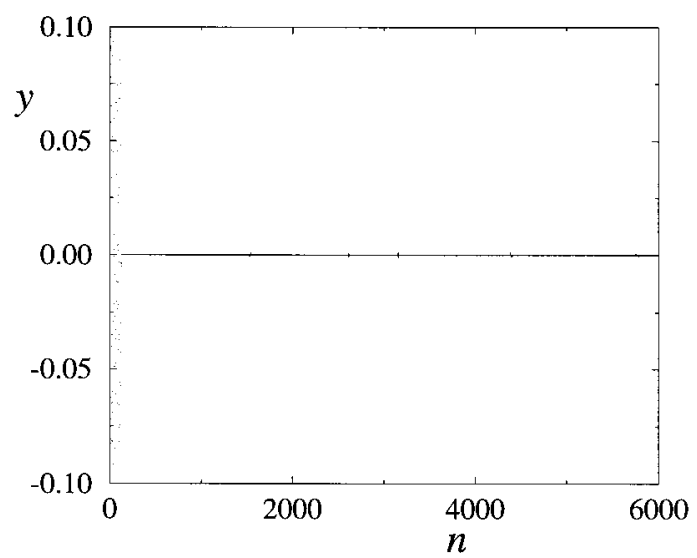

FIG. 7. A projection of the transverse dynamics under control.

tivated when iterates are within a distance of 0.1 of the period 2 point. A pair of perturbations are applied to the system when an iterate is within a distance of 0.01 of a period 2 point in order to place the iterate in the invariant subspace, thereby restoring chaotic behavior. Since iterates will never be placed precisely within the subspace, the state of the system will move away from synchronous behavior and when it does so, parameter perturbations are applied at the earliest opportunity in order to retain synchronous behavior. In Fig. 7 the transverse dynamics is shown when parameter perturbations are activated and the system brought to synchronous behavior. Thereafter, perturbations are applied when the dynamics begin to stray from the vicinity of the synchronous subspace and iterates fall within the vicinity of the period 2 orbit suitable for control. As can be seen from Fig. 7, very effective synchronization is possible using parameter perturbations.

\section{APPLICATIONS}

The coupled systems which we have considered all have a reflectional symmetry which derives from the two-way coupling. However, the methods could easily be adapted to deal with one-way coupling, in which case there is still an invariant subspace corresponding to the synchronized state but there is no longer any symmetry in the system. Using oneway coupling and adjusting a parameter in only one of the systems, this method could be used in the context of secure communication. There are various ways in which synchronization of chaotic systems can be used in this area (see, for example, the review of Ogorzalek [10]). One such method is to send two chaotic signals which correspond to either a zero or a one in a binary encoded message. The received signal can be fed into two different systems each of which will synchronize with only one of the two possible transmitted signals in order to determine which signal was sent. One possible drawback of this method is that the transient time before synchronization could be long so that sufficient time must be allowed for synchronization to occur.

A simple alternative to this method is to send two signals which do not naturally synchronize but can be made to synchronize by the use of parameter perturbations in the receiving system. Thus the two signals which are sent are chosen so that they will synchronize with parameter perturbations when coupled with the identical oscillator but with the property that parameter perturbations applied in the other case have the effect of moving iterates away from the invariant subspace. If a fixed or periodic point is chosen whose neighborhood is visited frequently by the chaotic motion and for which there is strong contraction towards the invariant subspace, then the transient time before synchronization can be significantly reduced, thus enabling a faster transmission of the signal.
[1] Y. S. Tang, A. I. Mees, and L. O. Chua, IEEE Trans. Circuits Syst. 30, 620 (1983).

[2] L. M. Pecora and T. C. Carroll, Phys. Rev. Lett. 64, 821 (1990).

[3] T. Yamada and H. Fujisaka, Prog. Theor. Phys. 70, 1240 (1983).

[4] S. Hayes, C. Grebogi, and E. Ott, Phys. Rev. Lett. 70, 3031 (1993).

[5] L. M. Kocarev and T. D. Stojanovski, IEICE Trans. Fund. Electron. Commun. Comput. Sci. 78, 1142 (1995).

[6] R. Roy and K. S. Thornburg, Phys. Rev. Lett. 72, 2009 (1994).

[7] Y. H. Yu, K. Kwak, and T. K. Lim, Opt. Quantum Electron 27, 535 (1995).

[8] Y. H. Yu, K. Kwak, and T. K. Lim, J. Korean Phys. Soc. 28, 109 (1995).

[9] K. M. Cuomo, A. V. Oppenheim, and S. H. Strogatz, IEEE Trans. Circuits Syst. II-Anal. Dig. Sig. Proc. 40, 626 (1993).
[10] M. J. Ogorzalek, IEEE Trans. Circuits Syst. I-Fund. Theor. Appl. 40, 693 (1993).

[11] Y-C. Lai and C. Grebogi, Phys. Rev. E 47, 2357 (1993).

[12] Y-C. Lai and C. Grebogi, Phys. Rev. E 50, 1894 (1994).

[13] Y. Nagai, X-D. Hua, and Y-C. Lai, Phys. Rev. E 54, 1190 (1996).

[14] P. Ashwin, J. Buescu, and I. Stewart, Phys. Lett. A 193, 126 (1994).

[15] P. Ashwin, J. Buescu, and I. Stewart, Nonlinearity 9, 703 (1996).

[16] P. Ashwin, Phys. Lett. A 209, 338 (1995).

[17] E. Ott, C. Grebogi, and J. A. Yorke, Phys. Rev. Lett. 64, 1196 (1990).

[18] P. J. Aston and M. Dellnitz, Int. J. Bifurcation Chaos 5, 1643 (1995).

[19] C. M. Bird, Ph.D. thesis, University of Surrey, 1996. 